\title{
Research of Rolling Shear Motion Laws Based On Improved PSO
}

\author{
Yugui Li and Yanxu Wei \\ School of Mechanical Engineering, Taiyuan University of Science and \\ Technology, Taiyuan 030024, China \\ liyugui2008@163.com,weiyanxu2012@126.com
}

\begin{abstract}
Making pure rolling shear as the target, based on the principles analysis of shear mechanism in the rolling shear, trajectories of the overlap and the horizontal displacement of the upper blade during the cutting process are directly calculated out. And then the trajectories are optimized with improved natural selection-based particle swarm optimization algorithm (selPSO) deemed lengths of the key rods as variables. On this basis, motion laws of the driving links are reversely worked out through solving position equations of the rolling shear's mechanism, which are nonlinear equations, with selPSO. The calculated results show that the synthetic horizontal translation (mean value of all horizontal displacement relating to calculated points) of the upper blade reduced by $34.96 \%$ and the overlap becomes a constant, which can realize the pure rolling cutting very well and improve the shear quality of the steel plate; selPSO is applicable to solve nonlinear equations in the project, which can overcome the problem of high sensitivity to initial value and improve the computational efficiency.
\end{abstract}

Keywords: rolling shear, selPSO, nonlinear equations, optimization, overlap, horizontal displacement

\section{Introduction}

The shear machine is a key equipment to ensure the shear quality of steel plate in steel plate finishing line. The hydraulic rolling shear ensures a rolling shear depending on the drive sequence and matching speeds of the hydraulic cylinder on its both sides. Compared with traditional shear machines, it omits the transmission mechanism such as motors, gearboxes and crankshafts, etc., which simplifies the equipment agency, reduces the energy consumption and improves the shear quality of the cross section [1]. However, the study on it is very hard due to its complex structure and heavy load [2].

In 1971, the rolling shear was developed for the first time in Germany, and then it was mastered by Japan, Russia and Australia etc., [3]. In china, it was studied by many steel factories and institutions; Qingxue. Huang and Lifeng. Ma in Taiyuan University of Science and Technology put forward the rod-optimized mathematical model of the rolling shear synthesized the accurate calculation of four positions, and the trajectory of the mechanism is simulated with computer [4-6]; Huixin. Yang in Beijing University of Science and Technology and Fuseng. Sun in Ma'anshan iron and steel Limited by Share Ltd establish the trajectory functions of arbitrary point in the upper blade deemed the crank angles as variables by analyzing the theory of the rolling shear's mechanism, and realize the pure rolling shear through the dynamic lowest points of the upper blade [7]; Kangkang. Li in Dalian University of Technology proposes a design method of rolling shear mechanism size which based on the mechanism cent rode to ensure the pure rolling shear, and the essence of this method is the problem of mechanism centrode path synthesis which is been solved by optimization method etc., [8-11]. However, the problems of the uneven overlap 
quantity as well as the horizontal displacement of the upper blade haven't been solved by any factory or institution now.

In this paper a new method is proposed that the theory trajectories of the dynamic lowest points and the midpoint in the cutting edge, associated with the overlap quantity and the horizontal displacement of the upper blade separately, are worked out under the fixed coordinate system according to the theory of rolling shear. Analyzing the mechanism of rolling shear, the actual trajectories of the dynamic lowest points are directly calculated out. A system of nonlinear equations is formed by setting the difference between the actual trajectory and the theoretical trajectory of the dynamic lowest points equal to zero, and selPSO is employed for the solutions. On this basis, the actual trajectory of the midpoint in the cutting edge can be worked out, whose difference with the theoretical trajectory is deemed as fitness value (optimization object) $G$; after that, selPSO is employed to optimize the lengths of the levers. Motion laws of the driving links are reversely worked out through solving position equations of the rolling shear's mechanism with selPSO.

One of the key problems is the solution of the position equations, which are nonlinear equations. Solving nonlinear equations is perhaps the most difficult problem in all of numerical computation. The convergence and performance characteristics are highly sensitive to the initial value of the solution for most numerical methods such as Newton's method. However, it is very difficult to select a reasonable initial value for most systems of nonlinear equations in the project. Aiming at these problems, the problem of solving nonlinear equations is transformed to an optimization problem, which is solved with selPSO. This method can efficiently overcome the problem of high sensitivity to the initial value of the solution, and improve the computational efficiency, which is very applicable to solve nonlinear equations in the project.

The calculated results show that the synthetic horizontal displacement of the upper blade decreases by $34.96 \%$ and the overlap turns into a constant, which can improve the shear quality of the steel plate and extend the life of the upper blade.

\section{Selpso and Solution of the Nonlinear Equations}

\section{1. selPSO}

Particle swarm optimization algorithm (PSO), found by Kennedy and Eberhart in 1995, is a kind of intelligent optimization algorithm originated from the study of birds seeking food [11], and then in order to promote the explorations in early optimization stages, the inertia weight $w$ is introduced into PSO [12]. Owing to its simple structure, PSO is developing rapidly and has plenty of modified forms, which is easy to understand and use [13-17].

A modified PSO brought forward by Shi are as follows:

$$
\begin{gathered}
v_{i}^{k+1}=w v_{i}^{k}+c_{1} r_{1}\left(\text { pbest }_{i}^{k}-x_{i}^{k}\right)+c_{2} r_{2}\left(\text { gbest }_{i}^{k}-x_{i}^{k}\right) . \\
x_{i}^{k+1}=x_{i}^{k}+v_{i}^{k+1} .
\end{gathered}
$$

Where equation (1) is to calculate the new velocity and equation (2) is to calculate the new position; $v$ represents the velocity and $x$ represents the position; $w$ is the inertia weight; $i$ is the ith particle and $k$ is the $k t h$ generation; $c 1$ and $c 2$ denote weighing factors called accelerated coefficients; $r 1$ and $r 2$ are random variables uniformly distributed within [0,1]; pbest $t_{i}$ denotes the ith personal best fitness and gbest $_{i}$ denotes the ith global best fitness; the initial velocity and position of each particle are random variables generated by the standard normal distribution. 
The natural selection-based particular swarm optimization algorithm is a combination of the particle swarm optimization algorithm and the theory of natural selection, which can obtain precise solutions. This method ranks particles according to the fitness value $G$, the worse half of which are replaced by the better half, and in the meantime, the pbest of each particle and the gbest are kept to go on the next iteration. In addition, the initial positions can be roughly selected according to practical requirement, and neglect iterations followed if the fitness value $G$ meets the standard (such as $G<R$ ), which improves calculation effectives. Thus the modified natural selection-based particle swarm optimization algorithm is obtained, the flow of which is shown in Figure 1.

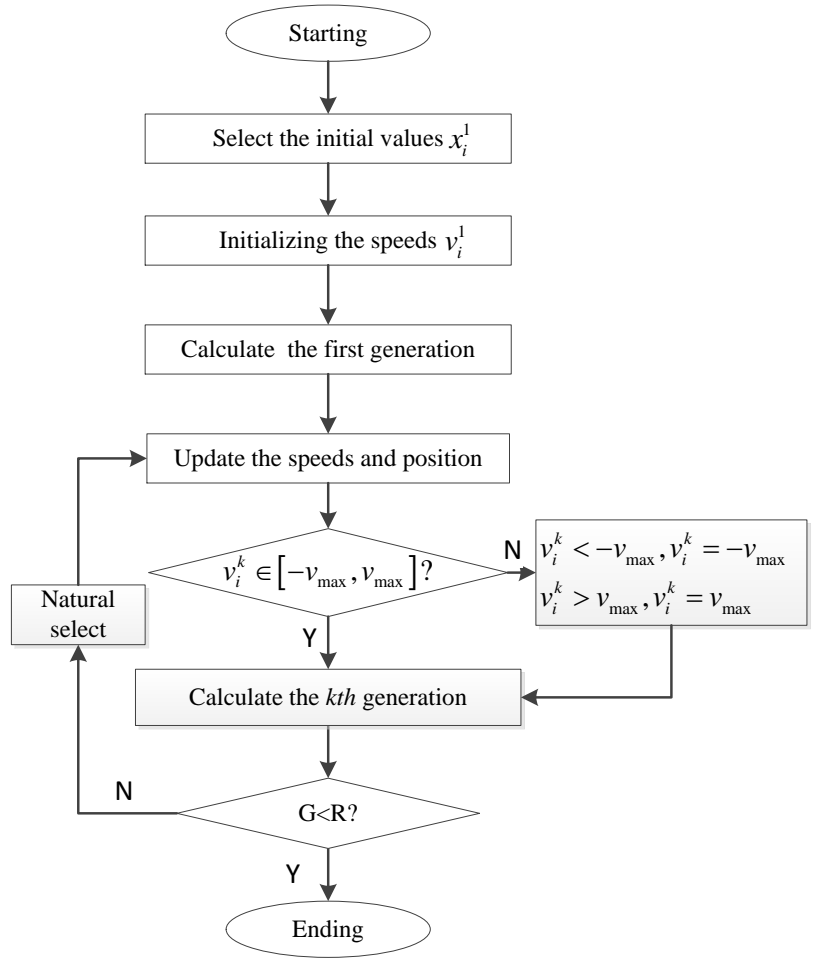

Figure 1. Calculation Flow Chart of selPSO

\subsection{The Solving of the Nonlinear Equations}

In the operation of the mechanism, variables are unpredictable owing to that they vary with the time and motion. Meanwhile, the convergence, convergence velocity and performance characteristics are highly sensitive to the initial value [18], which leads to difficulties when adopting most numerical methods. Aiming at this problem, selPSO, which can overcome the problem of high sensitivity to the initial value, is employed to solve the systems of nonlinear equations.

A system of the linear equations is as follows:

$$
F(x)=\left[f_{1}(x), f_{2}(x), f_{3}(x), L f_{n}(x)\right]^{T}=0
$$

Where $x=\left(x_{1}, x_{2}, x_{3}, \ldots x_{n}\right)^{T}$ are the $n$ variables;

Set the fitness value $G$ :

$G=\left|f_{1}(x)\right|+\left|f_{2}(x)\right|+\left|f_{3}(x)\right|+\cdots+\left|f_{n}(x)\right|$ Then solve the system of nonlinear equations with selPSO according to the Figure 1 . 


\section{Reverse Solving of the Motion Laws during the Stage of Cutting Rolling}

\subsection{Establishment of Vector Polygons}

As shown in Figure 2, the hydraulic rolling shear is the composition of four links, one guide rod, two blades and two hydraulic cylinders. According to the composition principle of planar mechanism, the hydraulic cylinders and the piston rods on both sides can be omitted, since they are assur groups. The simplified kinematic diagram of mechanism is shown as Figure 3.

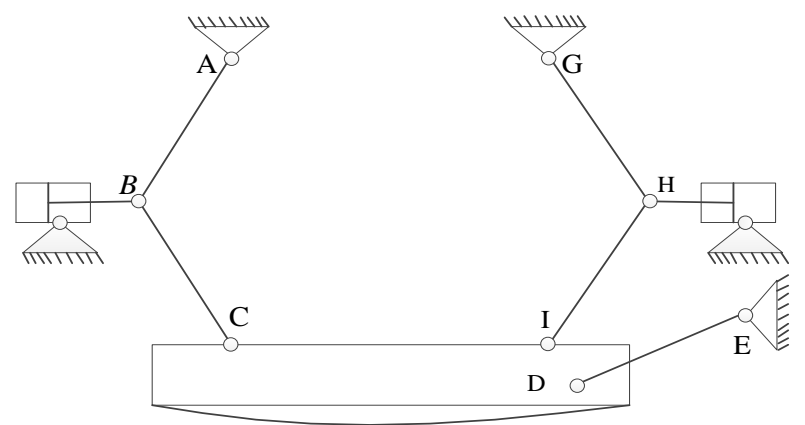

Figure 2. Kinematic Diagram of Mechanism of the Rolling Shear

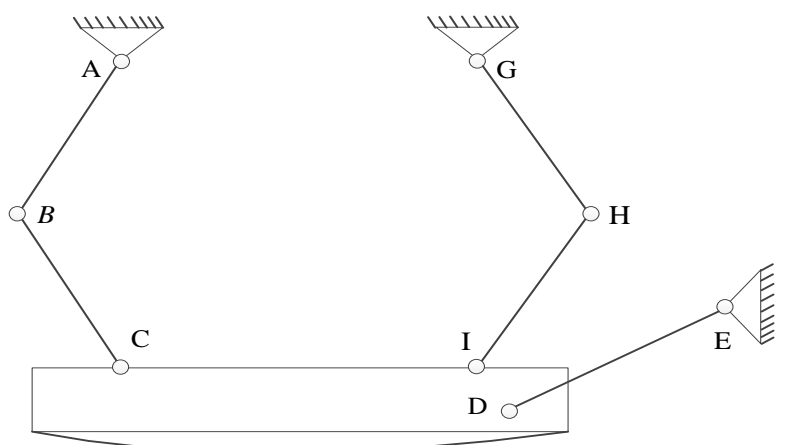

Figure 3. Simplified Kinematic Diagram of Mechanism of Rolling Shear

Formula of degrees of freedom is as follows:

$$
F=3 n-\left(2 p_{l}+p_{h}\right) .
$$

Where $n$ represents the moving links; $p l$ represents the lower pair; $p h$ denotes the higher pair. It can be seen from Figure 3 that the parameters of them are $n=6, p l=8, p h$ $=0$; the degrees of freedom $F$ are 2 calculated by the equation (3). The number of driving links, the lever $\mathrm{AB}$ and lever $\mathrm{GH}$, are equal to the degrees of freedom to ensure the definite movement.

As shown in Figure 4, two moving coordinate systems $x_{1} O y_{1}$ and $x_{2} D y_{2}$ and one fixed coordinate system $x E y$ are established, and the kinematic diagram of mechanism of the rolling shear is expressed by link vectors. 


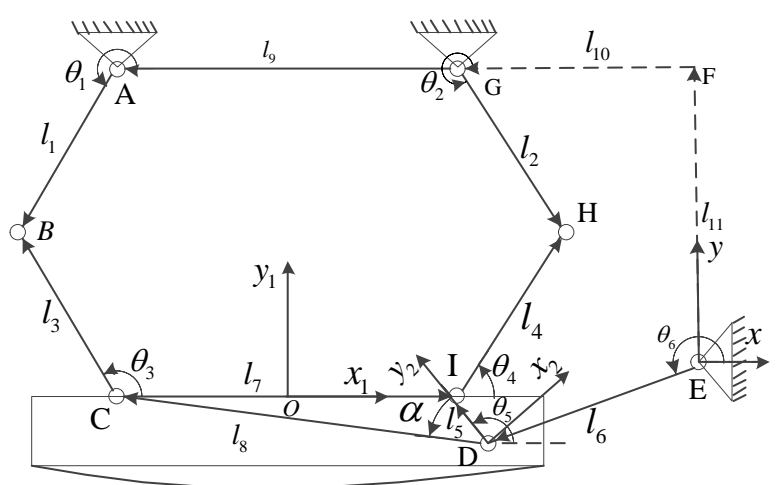

Figure 4. Kinematic Diagram of Mechanism

Where $\theta_{1} \in[\pi \square 2 \pi], \theta_{2} \in[\pi \square 2 \pi], \theta_{3} \in[0 \square \pi], \theta_{4} \in[0 \square \pi], \theta_{5} \in[0 \square \pi]$, $\theta_{6} \in[\pi \square 2 \pi]$.

\subsection{The Calculation of Dynamic Lowest Points and the Midpoint}

The dynamic lowest points are one of the most important parameters for the rolling shear, which directly determine the overlap quantity. During the process of rolling cut, if the overlap quantity is too large, the steel plate will be bending, while if the overlap quantity is too small, the steel plate will not be cut [1].

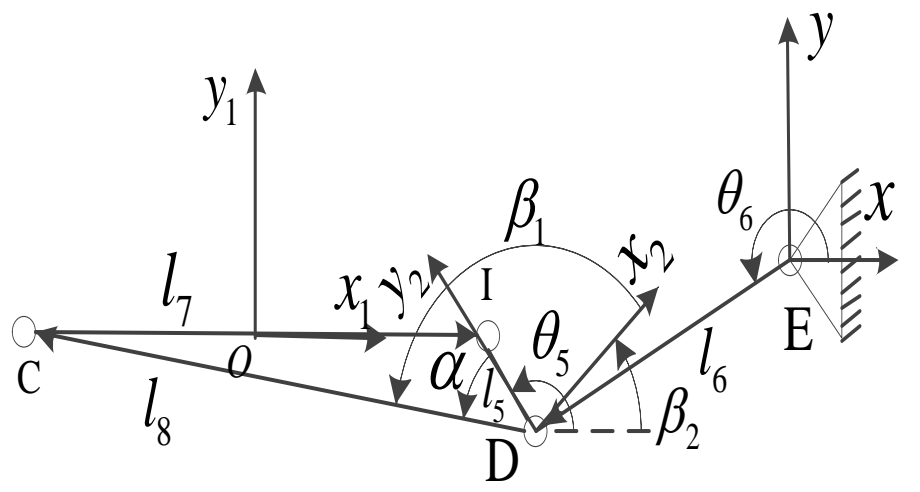

Figure 5. Parameters of the Rolling Shear

The transforming matrix $M_{12}$ from $x_{1} O y_{1}$ to $x_{2} d y_{2}$ and the transforming matrix $M_{20}$ from $x_{2} D y_{2}$ to $x d y$ are as follows:

$$
\begin{aligned}
& M_{12}=\left(\begin{array}{ccc}
\cos \beta_{2} & \sin \beta_{2} & d \cos \beta_{1} \\
-\sin \beta_{2} & \cos \beta_{2} & d \sin \beta_{1} \\
0 & 0 & 1
\end{array}\right) . \\
& M_{20}=\left(\begin{array}{ccc}
\cos \theta_{5} & \sin \theta_{5} & l_{6} \cos \theta_{6} \\
-\sin \theta_{5} & \cos \theta_{5} & l_{6} \sin \theta_{6} \\
0 & 0 & 1
\end{array}\right) .
\end{aligned}
$$


The relationship of the coordinate under the fixed coordinate system $x E y$ with the coordinate under the moving coordinate system $\mathrm{x} 1 \mathrm{Oy} 1$ is as followed:

$$
\left(\begin{array}{l}
x \\
y \\
1
\end{array}\right)=M_{20} M_{12}\left(\begin{array}{l}
x_{1} \\
y_{1} \\
1
\end{array}\right) .
$$

The height of the upper blade is $h$, so the coordinate of the midpoint is $(0,-h)$ under the moving coordinate system $x_{l} O y_{1}$, and then the coordinate of the midpoint under the fixed coordinate system is obtained through (4):

$$
\left\{\begin{array}{l}
x_{h} \\
y_{h} \\
1
\end{array}\right)=M_{20} M_{12}\left(\begin{array}{l}
0 \\
-h \\
1
\end{array}\right) .
$$

According to the Figure 6, the relationship of the dynamic lowest points with the middle point of the blade can be expressed as:

$$
\begin{aligned}
& x_{k}=x_{h}-R \times \sin \beta \\
& y_{k}=y_{h}+R \times(1-\cos \beta)
\end{aligned}
$$

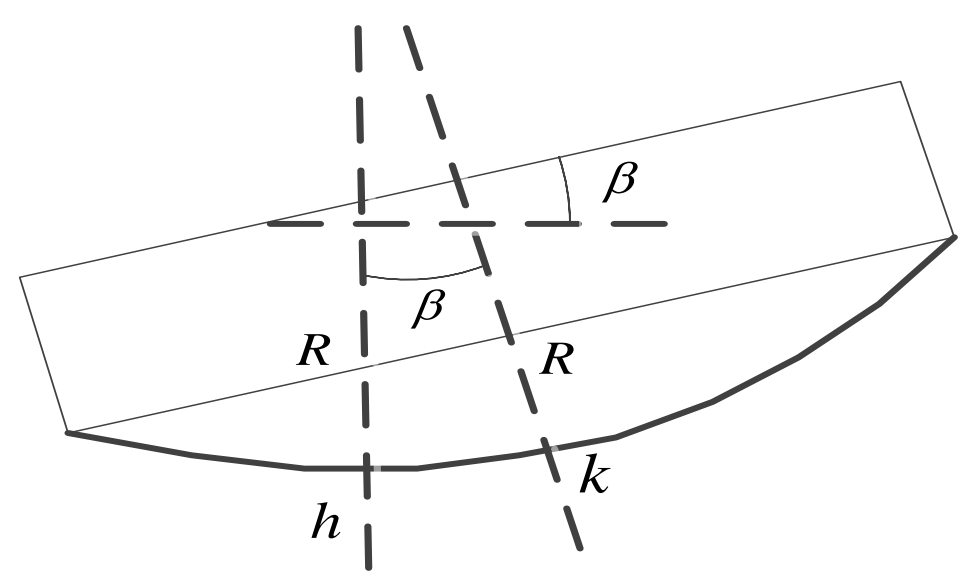

Figure 6. Relationship of the Dynamic Lowest Points with the Middle Point

It can be seen from Figure 4 and Figure 5 that the turning angle of the upper blade is $\beta=\theta_{5}-\beta_{2}$, and the dynamic lowest points can be obtained by (5) and (6):

$$
\left(\begin{array}{l}
x_{k} \\
y_{k} \\
1
\end{array}\right)=M_{20} M_{12}\left(\begin{array}{l}
0 \\
-h \\
1
\end{array}\right)+\left\{\begin{array}{l}
-R \times \sin \left(\theta_{5}-\beta_{2}\right) \\
R \times\left(1-\cos \left(\theta_{5}-\beta_{2}\right)\right) \\
1
\end{array}\right)
$$

The length of levers are known quantities and the $\theta_{5}$ and $\theta_{6}$ are unknowns during the motion of the mechanism. According to the requirement of the overlap, setting the upper blade rolling on the line $y=-s$, the expression of $x_{k}$ and $y_{k}$ cannot be calculated out, since (7) is a system of nonlinear equation, so the coordinate of the dynamic lowest points $x_{k}$ and $y_{k}$ must be taken on the line $\mathrm{y}=-\mathrm{s}$ in order. And then the $\theta_{5}$ and $\theta_{6}$ can be worked out through (7) with selPSO. 
According to the characteristics of the pure rolling, the trajectory of the midpoints is a curve, as the $1 \mathrm{~m}$ shown in Figure. 7. Its function can be expressed as:

$$
\begin{aligned}
& \theta=h u c h / r \\
& x=-h u c h+r \sin \theta+x_{0} . \\
& y=r(1-\cos \theta)+y_{0}
\end{aligned}
$$

Where huch is the arc length and $\theta$ is the corresponding angle.

The trajectory of the (8) is called trajectory 1.

To make the trajectory of midpoint close to trajectory 1 , the absolute value of the difference between the (5) and (8) is deemed as the fitness value during the process of optimizing the levers with selPSO.

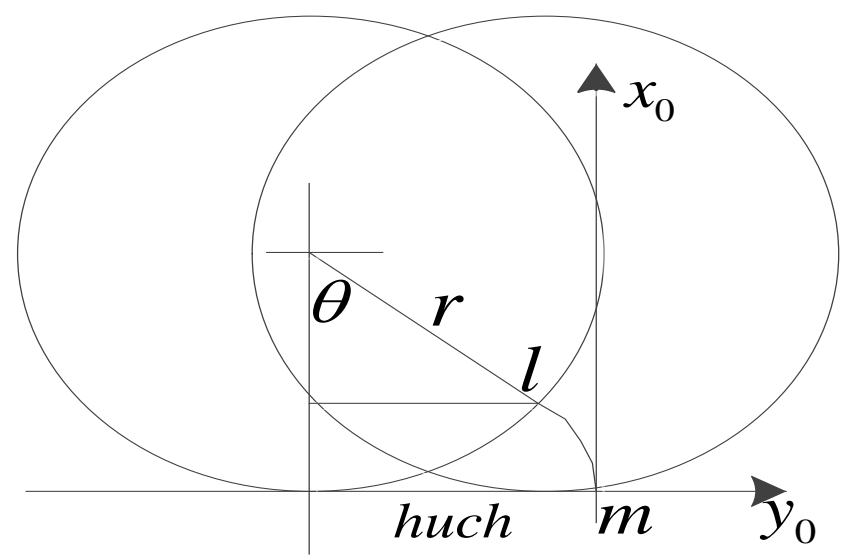

Figure 7. Trajectory of a Point on a Circle

\subsection{The Solving Of the Motion Laws of the Driving Links}

As shown in Figure 4 the lengths of the levers are the design constants, while the angles $\theta_{1}, \theta_{2}, \theta_{3}, \theta_{4}, \theta_{5}$ and $\theta_{6}$ are unknowns during the stage of rolling cut. Four equations are needed to solve the four unknowns $\theta_{1}, \theta_{2}, \theta_{3}, \theta_{4}$, since the $\theta_{5}$ and $\theta_{6}$ can be calculated out through (7). Here, two closed vector polygons are necessary for the four equations.

Setting the two closed vector polygons are ABCDOFG and OFGHIDO, the two closed position vector equations are express as:

$$
\begin{aligned}
& l_{1} \cos \theta_{1}-l_{3} \cos \theta_{3}-l_{8} \cos \left(\theta_{5}+\alpha\right)-l_{6} \cos \theta_{6}-l_{10}-l_{9}=0 \\
& l_{1} \sin \theta_{1}-l_{3} \sin \theta_{3}-l_{8} \sin \left(\theta_{5}+\alpha\right)-l_{6} \sin \theta_{6}+l_{11}=0 \\
& l_{2} \cos \theta_{2}-l_{4} \cos \theta_{4}-l_{5} \cos \theta_{5}-l_{6} \cos \theta_{6}-l_{10}=0 \\
& l_{2} \sin \theta_{2}-l_{4} \sin \theta_{4}-l_{5} \sin \theta_{5}-l_{6} \sin \theta_{6}+l_{11}=0
\end{aligned}
$$

It is very hard to select a reasonable initial value of (9), which is a system of nonlinear equations with four variables varying with the motion of the mechanism, and the unreasonable initial value may lead to long computing time, no convergence even wrong solutions for most numerical methods. Here, selPSO is employed to solve (9).

Assign a value to every equation: 


$$
\begin{aligned}
& b_{1}=l_{1} \cos \theta_{1}-l_{3} \cos \theta_{3}-l_{8} \cos \left(\theta_{5}+\alpha\right)-l_{6} \cos \theta_{6}-l_{10}-l_{9} \\
& b_{2}=l_{1} \sin \theta_{1}-l_{3} \sin \theta_{3}-l_{8} \sin \left(\theta_{5}+\alpha\right)-l_{6} \sin \theta_{6}+l_{11} \\
& b_{3}=l_{2} \cos \theta_{2}-l_{4} \cos \theta_{4}-l_{5} \cos \theta_{5}-l_{6} \cos \theta_{6}-l_{10} \\
& b_{4}=l_{2} \sin \theta_{2}-l_{4} \sin \theta_{4}-l_{5} \sin \theta_{5}-l_{6} \sin \theta_{6}+l_{11}
\end{aligned}
$$

Plus their absolute values as the fitness value:

$$
b=\left|b_{1}\right|+\left|b_{2}\right|+\left|b_{3}\right|+\left|b_{4}\right| \text {. }
$$

The $\theta_{1}$ and $\theta_{2}$, the motion laws of the driving links, can be solved through (9) with seIPSO.

The steps of solving the motion laws of the driving links and the optimization of the levers are as follows:

1. According to the requirement of the overlap, setting the upper blade rolling on the line $\mathrm{y}=-\mathrm{s}$, the coordinate of the dynamic lowest points $x_{k}$ and $y_{k}$ are taken on the line in order.

2. According to the $x_{k}$ and $y_{k}$ above, the corresponding $\theta_{5}$ and $\theta_{6}$ can be worked out through the solving of (7) with selPSO.

3. According to the $\theta_{5}$ and $\theta_{6}$ above, the levers $l_{1}, l_{3}, l_{5}, l_{6}$ and $l_{10}$ are optimized with selPSO deemed the synthetic horizontal translation, worked out by (5) and (6), as fitness value. The final $\theta_{5}$ and $\theta_{6}$ are worked out with the optimized $l_{1}, l_{3}, l_{5}, l_{6}$ and $l_{10}$ through the step (1) and (2).

4. After the corresponding parameters of the final $\theta_{5}$ and $\theta_{6}$ are worked out, the motion laws of the driving links $\theta_{1}$ and $\theta_{2}$ will be calculated by solving the (9) with selPSO.

\section{The Calculation Example}

The basic processing parameters are shown in Table 1.

\section{Table I. The Basic Processing Parameters}

\begin{tabular}{|c|c|}
\hline thickness $(\mathrm{mm})$ & $6 \sim 50$ \\
\hline width $(\mathrm{mm})$ & $1500 \sim 3200$ \\
\hline cutting times $(/ \mathrm{min})$ & 10 \\
\hline overlap $(\mathrm{mm})$ & 5 \\
\hline open degree $(\mathrm{mm})$ & 150 \\
\hline
\end{tabular}

The main parameters of the levers are shown in Table 2 . 
Table II. Type Sizes for Camera-Ready Papers

\begin{tabular}{|c|c|c|}
\hline variables & original results(mm) & $\begin{array}{c}\text { optimized } \\
\text { results(mm) }\end{array}$ \\
\hline 11 & 908 & 926.7 \\
\hline L3 & 908 & 689.3 \\
\hline L5 & 824 & 984.6 \\
\hline L6 & 960 & 809.3 \\
\hline 17 & 2400 & 2400 \\
\hline 110 & 2028 & 1704.3 \\
\hline
\end{tabular}

Figure 8 is the rolling shear in a factory.

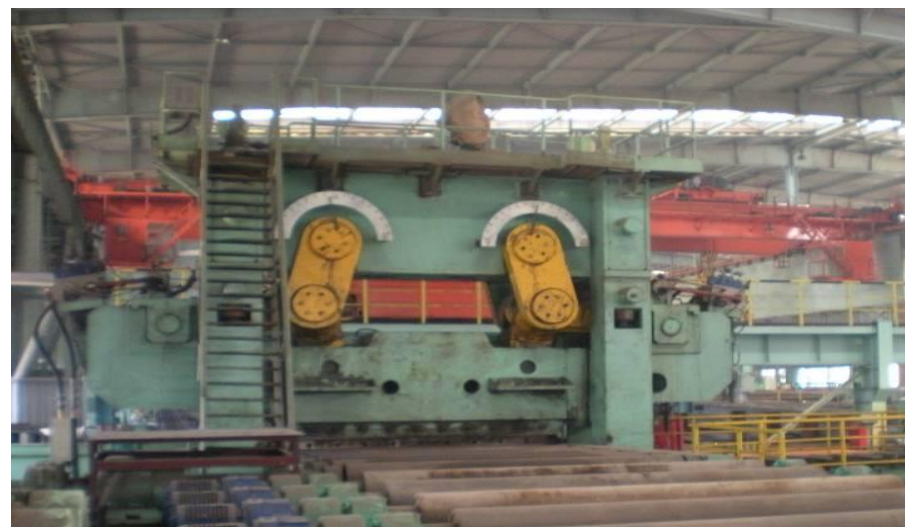

Figure 8. Picture of the Rolling Shear

It can be seen form Figure 9 that the optimized trajectory of dynamic lowest points becomes a horizontal line during the stage of rolling cut, which means the overlap becomes a constant. Figure 10 is the original trajectory of the midpoint and Figure 11 is the optimized trajectory of the midpoint during the stage of rolling cut. The results of calculation show that the optimized synthetic horizontal displacement of the midpoints decreases from 1.4341 to 0.9327 , reduced by $34.96 \%$.

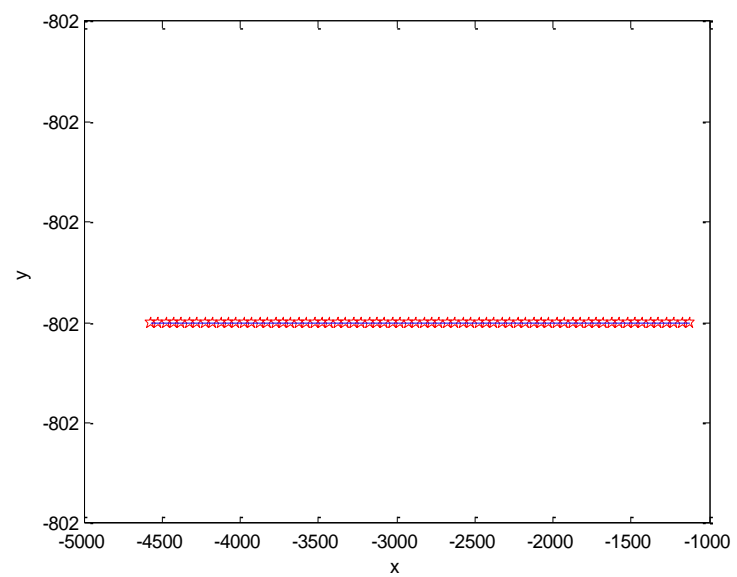

Figure 9. Optimized Trajectory of the Dynamic Lowest Points during the Stage of Rolling Cut 


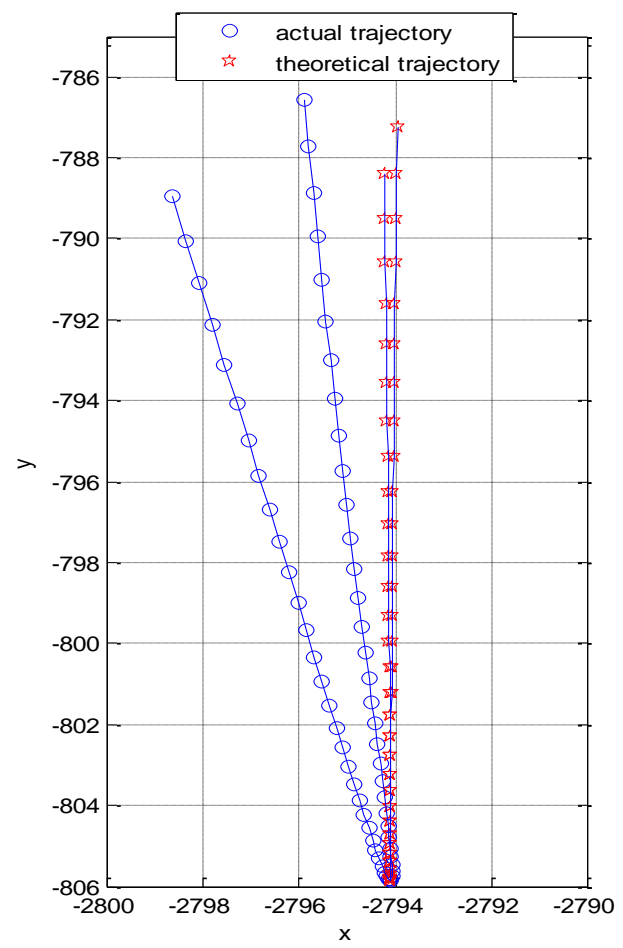

Figure 10. Oridinal Trajectory of the Midpoints During the Stage of Rolling

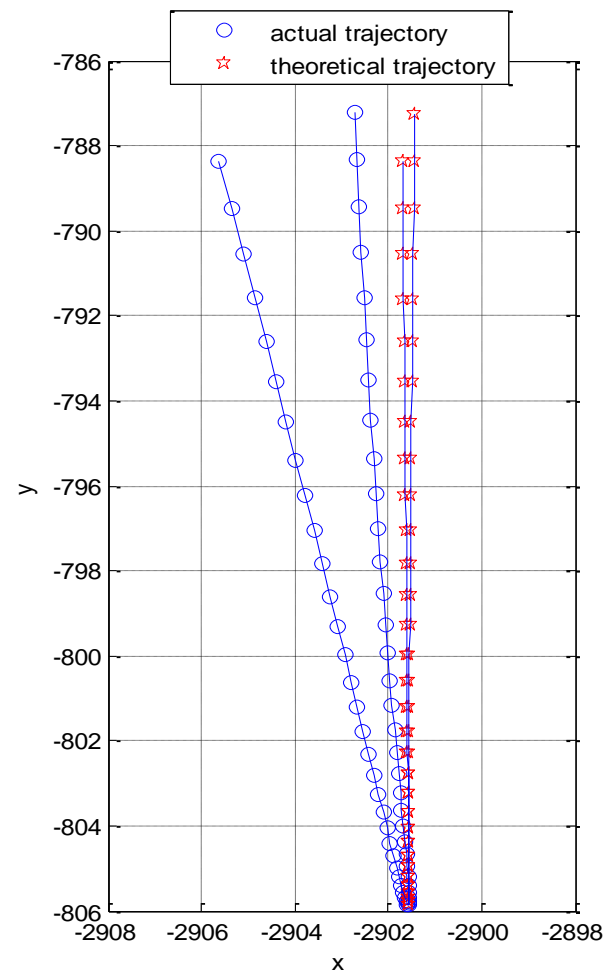

Figure 11. Optimized Trajectory of the Midpoints During the Stage of Rolling Cut

Figure 12 shows that the fitness values, under different initial values, vary with the number of iterations in the process of solving nonlinear equations with selPSO. It can be 
seen from Figure 11 that the fitness values under different initial guesses can approach to zero quickly, which proves selPSO can overcome the problem of high sensitivity to initial guess. If the fitness values meet the requirements (such as fitness values $\mathrm{G}<10-11$ ), the iterations followed can be omitted, thus improving the computational efficiency, and the mean time of solving nonlinear equations with selPSO is 0.321982 second.

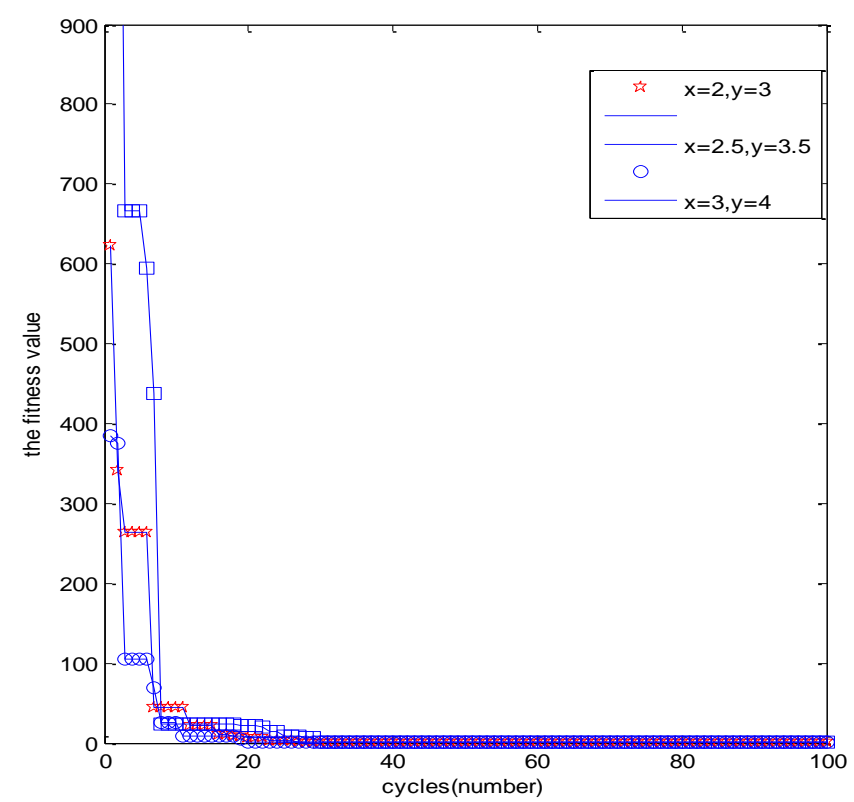

Figure 12. Variation of Different Fitness Values Under Different Initial Values

Figure 13 and Figure 14 are the motion laws of driving links.

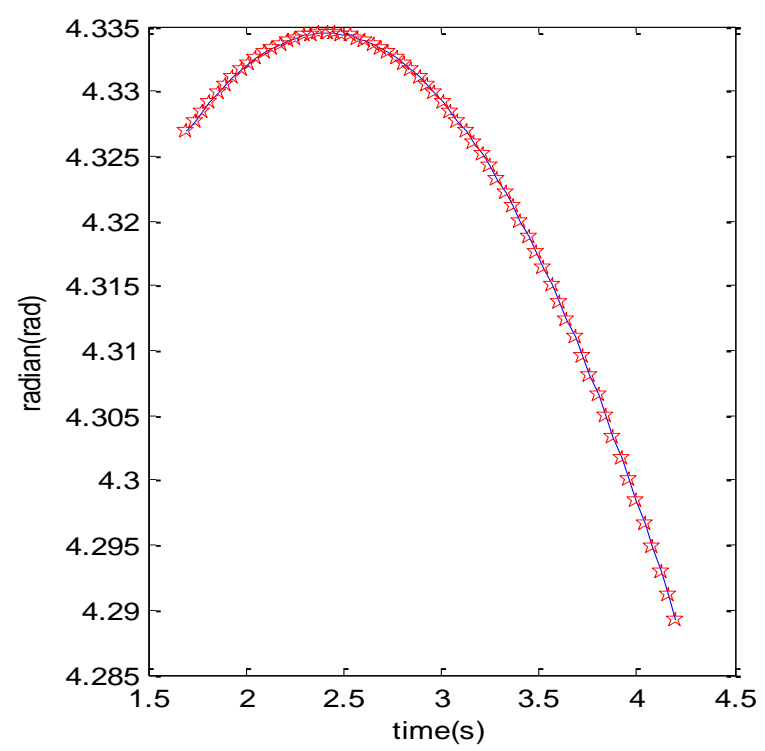

Figure 13. Optimized Motion Laws of $\theta_{1}$ During the Stage of Rolling Cut 


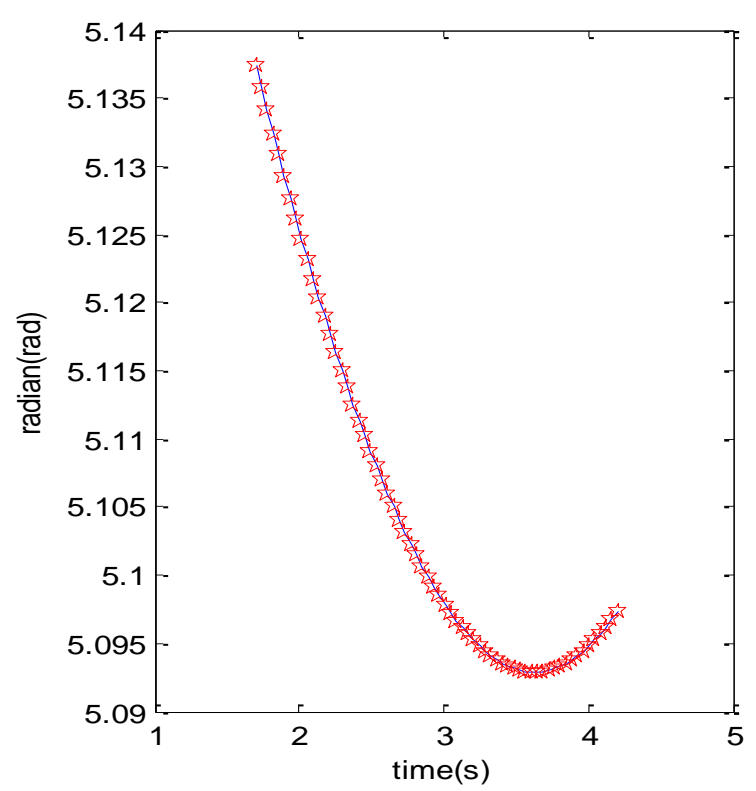

Figure 14. Optimized Motion Laws Of $\theta_{2}$ During the Stage of Rolling Cut

\section{Conclusions}

The motion laws of the driving links are worked out reversely through the movement trajectories of the dynamic lowest points and the midpoint in the cutting edge, which directly modifies the motion trajectory of the rolling shear in factories compared with the methods before. The synthetic horizontal displacement is decreased and the overlap quantity becomes a constant through this method, which can improve the shear quality of the steel plate and extend the life of the upper blade. Solving the systems of nonlinear equations with selPSO can efficiently overcome the problem of high sensitivity to the initial value, and improve the computational efficiency, which is very applicable to solve nonlinear equations in the project. The method in this paper is general, and can extend to a series of similar analysis.

\section{Acknowledgements}

The project is subsidized by The Research Project Supported by Shanxi Scholarship Council of China 2014-065 and Shanxi Province Natural Science Fund Project 2014011024-5.

\section{References}

[1] M. Lifeng, W. Gang, H. Qingxue, C. Zhibing, H. Zhiquan and H. Heyong, "Research on properties of compound linkage recapitulation rolling trace", Zhongguo Jixie Gongcheng (China Mechanical Engineering), vol. 24, no. 7, (2013), pp. 877-881.

[2] M. Masao and Y. Lu, "Precision cutting of sheets by means of a new shear based on rolling motion", Journal of materials processing technology, vol. 66, vol. 1 (1997), pp. 232-239.

[3] Y. N. Belobrov, Y. G. Bondar' and V. A. Plotnikov, "Reconstruction of the 5000 Plate Mill at Severstal", Metallurgist, vol. 47, no. 9, (2003), pp. 364-368.

[4] M. Lifeng, H. Qingxue, L. Ying, W. Jianmei and W. Xiaogang, "Establishment and application of mathematical model on spatial shear mechanism optimization of new-type steel rolling shear", JournalSichuan University Engineering Science Edition, vol. 40, no. 2, (2008), pp. 170.

[5] M. Lifeng, H. Qingxue, L. H. Zhiquan, C. Zhibing and T. Yaqin, "Establishment of Optimal Blade Clearance of Stainless Steel Rolling-Cut Shear and Test of Shearing Force Parameters", Journal of Iron and Steel Research, International, vol. 19, no. 9, (2012), pp. 52-61. 
[6] L. Yugui, M. Lifeng and H. Qingxue, "Research on rolling shear with single axle and asymmetric negative offset", Iron and Steel, vol. 43, no. 2, (2008), pp. 51-55.

[7] S. Fusen, Y. Huixin, Y. Xiaoqiang and Y. Deyin, "Simulation Research of Mechanism Parameter on Cut-to-Length Rolling Shear", Metallurgical Equipment, vol. 4, (2008), pp. 007.

[8] L. Kangkang, "Research on the Design Method of Rolling Shear Mechanism Based on Pure Rolling", MS thesis. Dalian University of Technology, (2009).

[9] H. Heyong, W. Jun, W. Jing, M. Lifeng and H. Qingxue, "Research on a new hydraulic rolling shear shearing mechanism and its control system", Proceedings of the Institution of Mechanical Engineers, Part B: Journal of Engineering Manufacture, 0954405414535590, (2014).

[10] H. Heyong, H. Qingxue, W. Jun and W. Jing, "The analysis of new hydraulic rolling shear servo system dynamic characteristics", Proceedings of the Institution of Mechanical Engineers, Part B: Journal of Engineering Manufacture, 0954405412472667, (2013).

[11] J. Kennedy, "Particle swarm optimization", Encyclopedia of Machine Learning. Springer US, (2010), pp. 760-766.

[12] Y. Shi and R. Eberhart, “A modified particle swarm optimizer”, Evolutionary Computation Proceedings, 1998. IEEE World Congress on Computational Intelligence. The 1998 IEEE International Conference on. IEEE, (1998).

[13] L. Zemin and W. Zong, "Complicated Graphics Model based on Neural Network of Particle Swarm", Journal of Multimedia, vol. 9, no. 3, (2014), pp. 333-339.

[14] T. Muling and Y. Jieming, "Improved Extraction Algorithm of Outside Dividing Lines in Watershed Segmentation Based on PSO Algorithm for Froth Image of Coal Flotation", Journal of Multimedia, vol. 9, no. 2, (2014), pp. 325-332.

[15] Z. Yu and Z. Honglei, "Image Stitching based on Particle Swarm and Maximum Mutual Information Algorithm”, Journal of Multimedia, vol. 8, no. 5, (2013), pp. 580-588.

[16] D. Zengfa and G. Lin, "Parameter Estimation of Conditional Random Fields Model by Improved Particle Swarm Optimizer", Journal of Computers, vol. 6, no. 8, (2011), pp. 1628-1633.

[17] W. Benzheng, Z. Zhimin and P. Xin, "Spatial information based medical image registration using mutual information", Journal of Multimedia, vol. 6, no. 3, (2011), pp. 236-243.

[18] H. Wang and L. Zhi-Shu, "Simpler and more effective particle swarm optimization algorithm", Ruan Jian Xue Bao, (Journal of Software), vol. 18, no. 4, (2007), pp. 861-868.

[19] L. Ya Zhong, Y. DuanCai and T. GuoJin, "Hybrid genetic algorithm for solving systems of nonlinear equations", Jisuan Lixue Xuebao (Chinese Journal of Computational Mechanics) (China), vol. 22, no. 1, (2005), pp. 109-114.

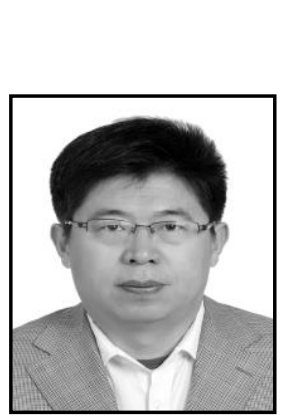

\begin{abstract}
Authors
Yu-Gui Li, born in Shanxi in 1967, is a professor of Taiyuan University of Science and Technology, and gained the doctor's degree at Beijing Institute of Technology in China, Beijing in 2007. $\mathrm{He}$ has authored or co-authored more than 20 publications collected by EI or SCI, and hold more than 10 Chinese patents. His achievements Granted with National 2nd class Progress Awards. Mr. $\mathrm{Li}$ is the executive director and the Deputy Secretary General of Shanxi Mechanical Engineering Society. He is also a leader of Shanxi Province young academic and the leader of Engineering Research Center for Department of Heavy Machinery Education, Taiyuan University of Science and Technology.
\end{abstract}

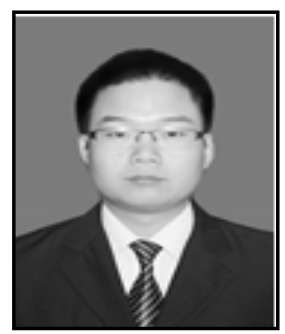

Yan-Xu Wei, born in Hebei in 1987, is a Master of Mechanical Engineering in the School of Mechanical Engineering at the Taiyuan University of Science and Technology. His current research interests include Fracture and Damage Mechanics, Computational Mechanics and Mechanical design. He has authored 2 publications. 
International Journal of Control and Automation Vol. 8, No. 6 (2015) 